\title{
The Reynolds analogy for the mixed convection over a vertical surface with prescribed heat flux
}

\section{Journal Article}

Author(s):

Magyari, E.; Pop, I.

Publication date:

2009

Permanent link:

https://doi.org/10.3929/ethz-b-000115241

Rights / license:

In Copyright - Non-Commercial Use Permitted

Originally published in:

Zeitschrift für angewandte Mathematik und Physik 60(2), https://doi.org/10.1007/s00033-008-7131-1 


\title{
The Reynolds analogy for the mixed convection over a vertical surface with prescribed heat flux
}

E. Magyari* and I. Pop

\begin{abstract}
The steady mixed convection boundary layer flow over a vertical surface with prescribed heat flux is revisited in this Note. The subset of solutions which can be obtained with the aid of the Reynolds analogy is discussed in a close relationship with the dual solutions reported by Merkin and Mahmood [1] for impermeable, and more recently by Ishak et al. [2], for permeable surfaces.
\end{abstract}

Mathematics Subject Classification (2000). 76R05, /6R10.

Keywords. Boundary layer, mixed convection, Reynolds analogy, dual solutions, suction/injection.

\section{Introduction}

The steady mixed convection boundary layer flow of an incompressible fluid over an impermeable or permeable vertical surface with prescribed temperature, or with prescribed heat flux is governed in the Boussinesq approximation by the continuity, momentum and energy equations, [1], [2],

$$
\begin{aligned}
& u_{x}+v_{y}=0 \\
& u u_{x}+v u_{y}=v u_{y y}+U U_{x}+g \beta\left(T-T_{\infty}\right) \\
& u T_{x}+v T_{y}=\alpha T_{y y}
\end{aligned}
$$

where the subscripts denote partial derivatives with respect to the coordinates $x$ and $y$, and $U(x)=U_{0} \cdot(x / L)^{m}$ is the free stream velocity. The boundary conditions adopted are [1], [2],

$$
\begin{gathered}
\left.u\right|_{y=0}=0,\left.\quad v\right|_{y=0}=v_{0} \cdot\left(\frac{x}{L}\right)^{\frac{m-1}{2}},\left.\quad u\right|_{y \rightarrow \infty}=U(x) \\
-\left.k \frac{d T}{d y}\right|_{y=0}=q_{0} \cdot\left(\frac{x}{L}\right)^{\frac{5 m-3}{2}},\left.\quad T\right|_{y \rightarrow \infty}=T_{\infty} .
\end{gathered}
$$

\footnotetext{
* Corresponding author.
} 
With the aid of the stream function $\psi\left(u=\psi_{y}, v=-\psi_{x}\right)$ and the similarity transformations,

$$
\begin{aligned}
\psi & =\sqrt{v L U_{0}}\left(\frac{x}{L}\right)^{\frac{m+1}{2}} f(\eta), \quad \eta=\sqrt{\frac{U_{0}}{v L}}\left(\frac{x}{L}\right)^{\frac{m-1}{2}} y \\
T & =T_{\infty}+\Delta T\left(\frac{x}{L}\right)^{2 m-1} \theta(\eta) .
\end{aligned}
$$

Eqs. (1)-(4) reduce to the system of ordinary differential equations

$$
\begin{aligned}
& f^{\prime \prime \prime}+\frac{m+1}{2} f f^{\prime \prime}+m\left(1-f^{\prime 2}\right)+\lambda \theta=0 \\
& \frac{\theta^{\prime \prime}}{P r}+\frac{m+1}{2} f \theta^{\prime}+(1-2 m) f^{\prime} \theta=0
\end{aligned}
$$

subject to the boundary conditions

$$
\begin{gathered}
f(0)=f_{0}, \quad f^{\prime}(0)=0, \quad f^{\prime}(\infty)=1 \\
\theta^{\prime}(0)=-1, \quad \theta(\infty)=0 .
\end{gathered}
$$

In the above equations $L, \Delta T=\left(q_{0} / k\right)\left(v L / U_{0}\right)^{1 / 2}$ and $U_{0}$ specify the length, temperature and velocity scales, respectively, the primes denote differentiations with respect to the similarity variable $\eta$, and $\lambda=G r / R e^{2}$ is the mixed convection parameter with $G r=g \beta \Delta T L^{3} / v^{2}$, and $R e=U_{0} L / v$ the Grashof and Reynolds numbers, respectively. The other notations, as well as the choice of the coordinate system coincide with those of [1] and [2].

In References [1] and [2], the structure of solution space of the boundary value problem $(7)-(10)$ has been investigated for impermeable $\left(f_{0}=0\right)$ and both impermeable and permeable $\left(f_{0} \neq 0\right)$ surfaces, respectively. The main issue of the present Note is to discuss the subset of solutions of this boundary value problem which can be obtained with the aid of the Reynolds analogy.

\section{The Reynolds analogy}

The Reynolds analogy of the momentum and energy balance equations is based on the simple observation that for a uniform free stream $\left(m=0\right.$, i.e., $\left.U_{x}=0\right)$ and in the absence of buoyancy forces, Eqs. (2) and (3) possess for $\operatorname{Pr}=1$ (i.e., for $v=\alpha$ ) the same form for $u$ and $T$, i.e. Eqs. (2) and (3) reduce in fact to a single equation only. Consequently, for certain velocity and temperature boundary conditions, there always exists a temperature solution which is a linear function of streamwise velocity $u$.

This idea can also be extended for the case of the full Eqs. (2) and (3), with the distinction that the coefficients of the linear function mentioned should firstly be taken as functions of the streamwise coordinate $x$. Indeed, writing $T=A(x) u+$ $B(x)$, the asymptotic conditions $\left.u\right|_{y \rightarrow \infty}=U(x)$ and $\left.T\right|_{y \rightarrow \infty}=T_{\infty}$ immediately 
imply

$$
T=T_{\infty}+A(x)(u-U) .
$$

Substituting Eq. (11) in Eqs. (2) and (3) (with $\alpha=v$ ), one easily sees that the resulting equations become identical when $A=$ const. $\equiv A_{0}$, and $\left(U_{x}-g \beta A_{0}\right)(U-$ $u)=0$. Having in mind that $U=U_{0} \cdot(x / L)^{m}$, the latter condition requires $m=1$ and implies further $A_{0}=U_{0} /(g \beta L)$. Accordingly, Eq. (11) becomes

$$
T=T_{\infty}+\frac{U_{0}^{2}}{g \beta L}\left(\frac{u}{U_{0}}-\frac{x}{L}\right)=T_{\infty}+\frac{\Delta T}{\lambda}\left(\frac{u}{U_{0}}-\frac{x}{L}\right)
$$

and, as a consequence of this relationship, Eqs. (2) and (3) reduce to the same form

$$
u u_{x}+v u_{y}=v u_{y y}+\frac{U_{0}}{L} u .
$$

Furthermore, Eqs. (5) and (6) give for $m=1$

$$
u=U_{0} \frac{x}{L} f^{\prime}(\eta), \quad v=-\sqrt{\frac{U_{0} v}{L}} f(\eta), \quad T=T_{\infty}+\Delta T \frac{x}{L} \theta(\eta)
$$

and thus equations (12) and (13) become

$$
T=T_{\infty}+\Delta T \frac{x}{L} \frac{f^{\prime}(\eta)-1}{\lambda},
$$

and

$$
f^{\prime \prime \prime}+f f^{\prime \prime}+f^{\prime}\left(1-f^{\prime}\right)=0
$$

respectively. Comparing the third Eq. (14) to Eq. (15), one obtains that (within the Reynolds analogy) the similar temperature and velocity fields $\theta$ and $f^{\prime}$ corresponding to $m=1$ and $\operatorname{Pr}=1$ are related to each other by the simple relationship

$$
\theta(\eta)=\frac{f^{\prime}(\eta)-1}{\lambda} \quad \text { (Reynolds analogy). }
$$

\section{Solution and discussion}

As shown above, for the subset of solutions $\left\{f^{\prime}, \theta\right\}=\left\{f^{\prime},\left(f^{\prime}-1\right) / \lambda\right\}$ given by the Reynolds analogy only the single Eq. (16) has to be solved, instead of the system of coupled Eqs. (7) and (8). The corresponding solutions describe the mixed convection stagnation point flow $(m=1)$ of a fluid with Prandtl number $\operatorname{Pr}=1$. The goal of the present Section is the investigation of the corresponding boundary value problem (16), (9) in detail.

We first notice that Eqs. (17) and (10) yield for the dimensionless wall temperature $\theta(0)$ and skin friction $f^{\prime \prime}(0)$ the simple relationships

$$
\theta(0)=-\frac{1}{\lambda} \text { and } f^{\prime \prime}(0)=-\lambda .
$$




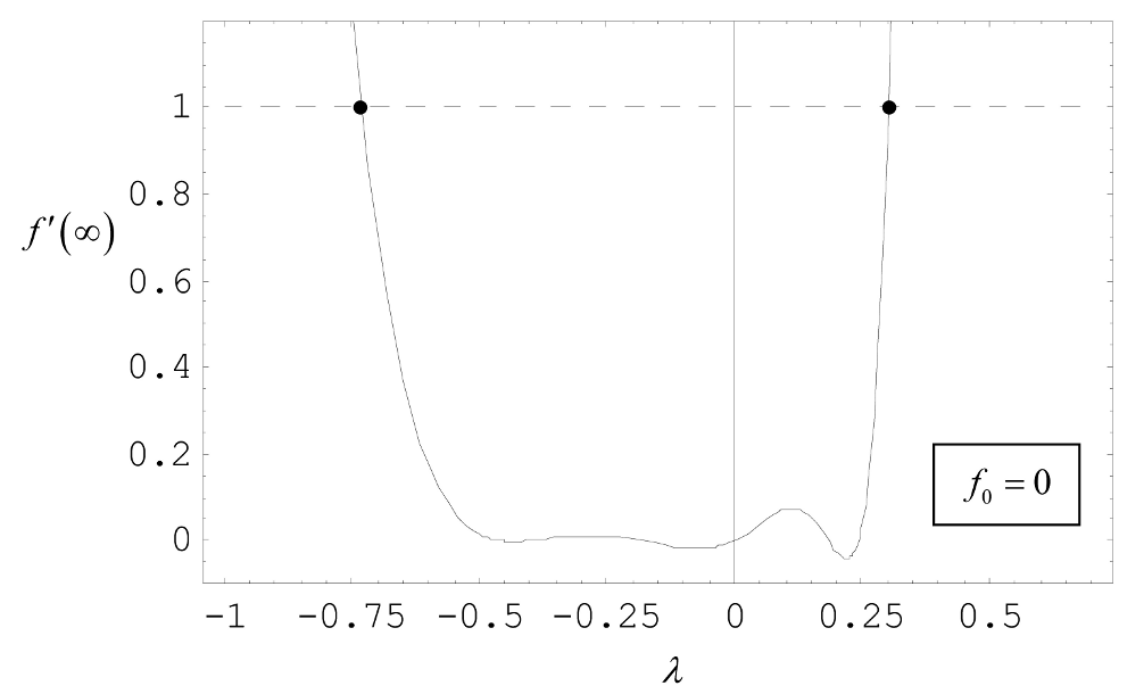

Figure 1. Plot of $f^{\prime}(\infty) \simeq f^{\prime}(10)$ as a function of $\lambda=-f^{\prime \prime}(0)$ for $f_{0}=0$. The two intersection points of the curve $f^{\prime}(10)$ with the horizontal line 1 (marked by dots) correspond to the $\lambda$-values $\lambda_{1}=-0.7314105$ and $\lambda_{2}=+0.3026979$, respectively. The associated similar velocity profiles $f^{\prime}(\eta)$ are plotted in Figure 2.

Therefore, the task is to find the values of the mixed convection parameter $\lambda$, for which the solution of the initial value problem

$$
\begin{gathered}
f^{\prime \prime \prime}+f f^{\prime \prime}+f^{\prime}(1-f)=0, \\
f(0)=f_{0}, \quad f^{\prime}(0)=0, \quad f^{\prime \prime}(0)=-\lambda
\end{gathered}
$$

satisfies (for a specified value of $f_{0}$ ) the asymptotic condition $f^{\prime}(-\infty)=1$.

Being faced here with an initial value problem with an additional condition, the solution is easily found. The procedure is illustrated in Figure 1 where $f^{\prime}(\infty) \cong$ $f^{\prime}(10)$ has been plotted as a function of $\lambda$ for $f_{0}=0$ (impermeable surface), and this curve has been intersected with the straight line $f^{\prime}(10)=1$ according to the asymptotic condition. Two intersection points of the curve $f^{\prime}(10)$ with the horizontal line 1 were found, namely at $\lambda_{1}=-0.7314105$ and $\lambda_{2}=+0.3026979$, respectively. The procedure has then to be repeated subsequently for $f^{\prime}(12)$, $f^{\prime}(15)$, etc. until the values of $\lambda_{1}$ and $\lambda_{2}$ do not change within the desired precision. These solutions, corresponding to $\left(f_{0}, \lambda\right)=\left(0, \lambda_{1}\right)$ and $\left(f_{0}, \lambda\right)=\left(0, \lambda_{2}\right)$ are plotted in Figure 2. The results of similar calculations performed for $f_{0}=-0.1$ (injection) and $f_{0}=+0.1$ (suction) are collected (together with the case $f_{0}=0$ ) in Table 1 .

We see that the left and right intersection points of the curve $f^{\prime}(\infty)$ with the straight line $f^{\prime}(\infty)=1$, having for a given $f_{0}$ the respective abscisses $\lambda_{\text {Left }}\left(f_{0}\right)<0$ and $\lambda_{\text {Right }}\left(f_{0}\right)>0$, specify two solution branches of the boundary value problem (16), (9). In the asymptotic limit $f_{0} \rightarrow \infty$ of large suction velocities, the de- 


\begin{tabular}{|c|c|c|c|c|}
\hline & $\lambda$ & $f^{\prime \prime}(0)$ & $\theta(0)$ & Branch \\
$f_{0}$ & Left/Right & Left/Right & Left/Right & $f^{\prime \prime}(0) ; \theta(0)$ \\
\hline 0.1 & -0.664197 & +0.664197 & +1.505577 & $U ; L$ \\
\hline 0 & +0.2504476 & -0.2504476 & -3.9928512 & $L ; L$ \\
\hline+0.7314105 & +0.7314105 & +1.3672212 & $U ; L$ \\
\hline & +0.3026979 & -0.3026979 & -3.3036238 & $L ; L$ \\
\hline & -0.8006085 & +0.8006085 & +1.2490499 & $U ; L$ \\
\hline
\end{tabular}

Table 1. The left and right values of $\lambda, f^{\prime \prime}(0)$ and $\theta(0)$ corresponding to three specified values of the suction/injection parameter $f_{0}$. The symbols $U$ and $L$ refer to the upper and lower branch of solutions according to Figures 1 and 3 of Reference [2], respectively.

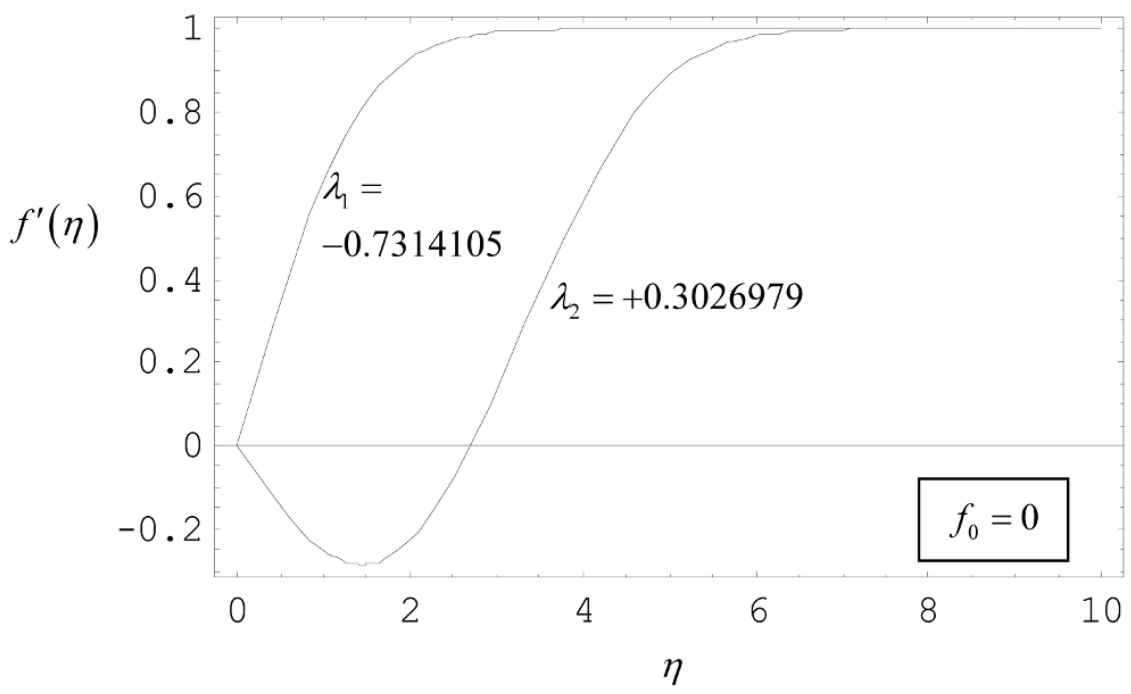

Figure 2. Plot of similar velocity profiles $f^{\prime}(\eta)$ corresponding to the skin frictions $f^{\prime \prime}(0)=-\lambda_{1}=0.7314105$ and $f^{\prime \prime}(0)=-\lambda_{2}=-0.3026979$, respectively, associated with the same value $f_{0}=0$ of the suction/injection parameter. 


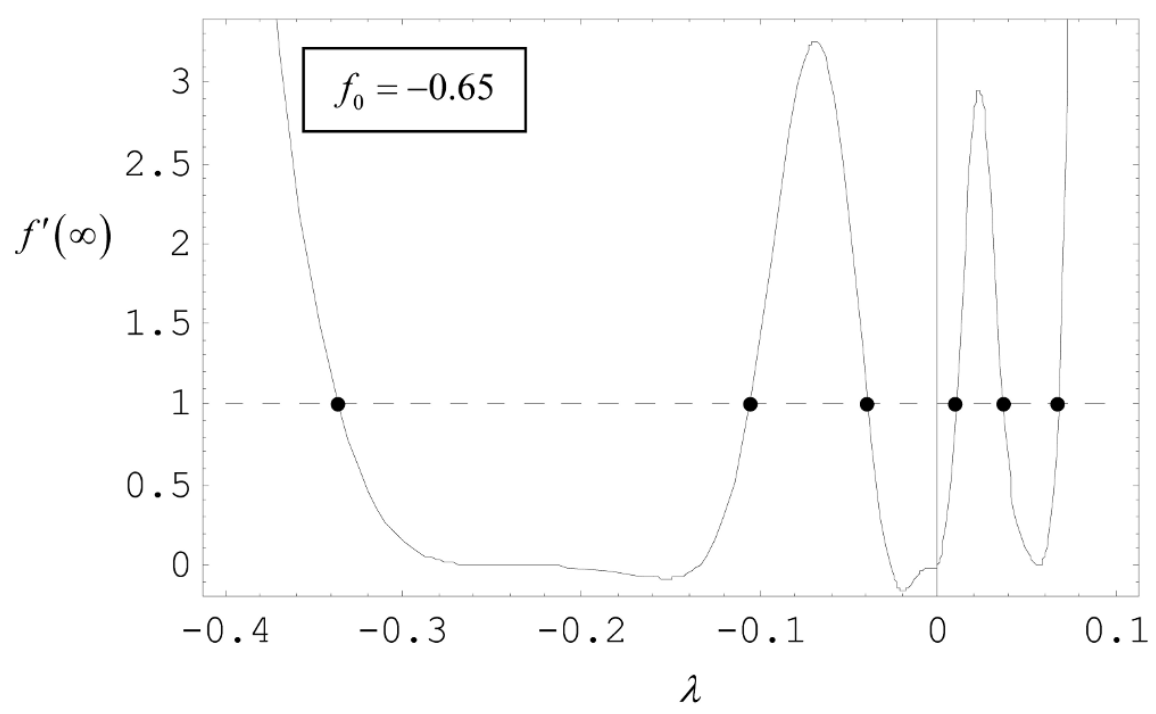

Figure 3. Plot of $f^{\prime}(\infty) \simeq f^{\prime}(13)$ as a function of $\lambda$ for $f_{0}=-0.65$. The six points of the curve $f^{\prime}(13)$ with the horizontal line 1 (marked by dots) correspond to the $\lambda$-values $\lambda_{1}=-0.3369764$, $\lambda_{2}=-0.1056770, \lambda_{3}=-0.0393022, \lambda_{4}=+0.0098145, \lambda_{5}=+0.0370746$ and $\lambda_{6}=+0.0676765$ respectively. The associated similar velocity profiles $f^{\prime}(\eta)$ are plotted in Figure 4.

pendence of the corresponding skin friction coefficients $f^{\prime \prime}(0)=-\lambda$ on $f_{0}$ can be estimated as follows:

In the case of the left branch we first substitute in Eqs. (16) and (9)

$$
\eta=\frac{\xi}{f_{0}}, \quad f(\eta)=f_{0}+\frac{F(\xi)}{f_{0}} \quad \text { (Left branch). }
$$

This substitution transforms Eqs. (16) and (9) in

$$
\begin{aligned}
& \dddot{F}+\left(1+\frac{F}{f_{0}^{2}}\right) \ddot{F}+\frac{\dot{F}(1-\dot{F})}{f_{0}^{2}}=0, \\
& F(0)=0, \quad \dot{F}(0)=0, \quad \dot{F}(\infty)=1
\end{aligned}
$$

where the dot denotes differentiation with respect to the scaled variable $\xi$.

The transformed Eqs. (21) suggest the power series expansion of the form

$$
F(\xi)=F_{0}(\xi)+\frac{F_{1}(\xi)}{f_{0}^{2}}+\ldots
$$

In this way, we obtain to the zeroth and the first order approximations in $1 / f_{0}^{2}$ the linear boundary value problems

$$
\begin{gathered}
\dddot{F}_{0}+\ddot{F}_{0}=0, \\
F_{0}(0)=0, \quad \dot{F}_{0}(0)=0, \quad \dot{F}_{0}(\infty)=1
\end{gathered}
$$


and

$$
\begin{gathered}
\dddot{F}_{0}+\ddot{F}_{1}=-F_{0} \ddot{F}_{0}-\dot{F}_{0}\left(1-\dot{F}_{0}\right), \\
F_{0}(0)=0, \quad \dot{F}_{0}(0)=0, \quad \dot{F}_{0}(\infty)=0 .
\end{gathered}
$$

As expected, the zeroth order approximation (23) yields the asymptotic suction profile

$$
\dot{F}_{0}=1-e^{-\xi}, \quad F_{0}=\xi-1+e^{-\xi} .
$$

For the solution of the first order correction (24) we obtain by elementary calculations

$$
F_{1}=2-\left(2+2 \xi+\frac{1}{2} \xi^{2}\right) e^{-\xi}
$$

such that

$$
F(\xi)=\xi-1+e^{-\xi}+\frac{1}{f_{0}^{2}}\left[2-\left(2+2 \xi+\frac{1}{2} \xi^{2}\right) e^{-\xi}\right] .
$$

Accordingly, the skin friction coefficient $f^{\prime \prime}(0)=f_{0} \ddot{F}(0)$ of the left solution branch results as

$$
\left.f^{\prime \prime}(0)=f_{0}+\frac{1}{f_{0}}+\ldots \quad \text { (Left branch, } f_{0} \rightarrow \infty\right) .
$$

In the case of the right branch, it is convenient to substitute in Eqs. (16) and (9)

$$
\eta=\frac{\xi}{f_{0}}, \quad f(\eta)=f_{0} \phi(\xi) \quad \text { (Right branch) }
$$

This substitution transforms Eqs. (16) and (9) in

$$
\begin{gathered}
\dddot{\phi}+\phi \ddot{\phi}-\dot{\phi}^{2}+\frac{1}{f_{0}^{2}} \dot{\phi}=0, \\
\phi(0)=1, \quad \dot{\phi}(0)=0, \quad \dot{\phi}(\infty)=\frac{1}{f_{0}^{2}} .
\end{gathered}
$$

The transformed Eqs. (30) suggest again a power series expansion of the form

$$
\phi(\xi)=\phi_{0}(\xi)+\frac{\phi_{1}(\xi)}{f_{0}^{2}}+\ldots
$$

However, in contrast to the left branch, in the leading order approximation the problem (30) is still non-linear

$$
\begin{gathered}
\dddot{\phi}_{0}+\phi_{0} \ddot{\phi}_{0}-\dot{\phi}_{0}^{2}=0, \\
\phi_{0}(0)=1, \quad \dot{\phi}_{0}(0)=0, \quad \dot{\phi}_{0}(\infty)=0 . \quad\left(f_{0} \rightarrow \infty\right)
\end{gathered}
$$

This problem could be solved only numerically, yielding for the scaled skin friction coefficient the value $\ddot{\phi}_{0}(0)=-0.06$. Consequently, we arrive at the result that, to the leading order, the skin friction coefficient $f^{\prime \prime}(0)=f_{0}^{3} \ddot{\phi}(0)$ of the right solution branch scales for large suction velocities as

$$
f^{\prime \prime}(0)=-0.04 f_{0}^{3}+\ldots \quad\left(\text { Right branch, } f_{0} \rightarrow \infty\right) .
$$


The higher order approximations can also be determined only numerically in this case. It is worth mentioning here that for the left branch the leading order approximation $f^{\prime \prime}(0) \cong f_{0}$ obtained from Eq. (28), gives a quite accurate result already for $f_{0}=10$, namely $f^{\prime \prime}(0)=10$, compared to the "exact" numerical result $f^{\prime \prime}(0) \cong 10.0976$. For the right branch by contrast, the leading order approximation (33) gives an accurate result only around $f_{0}=10^{4}$, namely $f^{\prime \prime}(0)=-4 \cdot 10^{10}$, compared to the "exact" numerical result $f^{\prime \prime}(0)=-4.0779 \cdot 10^{10}$.

The left and right branches discussed above can be continued also for negative values of $f_{0}$, corresponding to a lateral injection of the fluid. A qualitative analysis of the boundary value problem (16), (9) shows that the skin friction coefficient $f^{\prime \prime}(0)$ goes to zero smoothly for both solution branches as $f_{0} \rightarrow \infty$. However, the decrease of $f^{\prime \prime}(0)$ is very fast, so that it can be followed by numerical calculations to limited values of $f_{0}<0$ only. Namely, one obtains $f_{\text {Left }}^{\prime \prime}(0)=0.001636$ and $f_{\text {Right }}^{\prime \prime}(0)=-0.000024$ already at $f_{0}<-1.8$. At $f_{0}<-1.9$, the left value reduces to $f_{\text {Left }}^{\prime \prime}(0)=0.000076$ and the right one is not viewable any more. This behaviour, however, is not the only peculiarity of the injection case. In contrast to the increasing suction, with increasing lateral injection, also the shape of the curve $f^{\prime}(\infty)$ shown in Figure 1 changes dramatically, becoming more and more complex. A cascade of bifurcations, leading to further solution branches of the boundary value problem (16), (9) with both positive and negative values of the skin friction coefficient $f^{\prime \prime}(0)$ occur. This feature is illustrated in Figure 3 where $f^{\prime}(\infty) \cong f^{\prime}(13)$ has been plotted as function of $\lambda$ for $f_{0}=-0.65$. In this case six intersection points of the curve $f^{\prime}(13)$ with the horizontal line 1 were found, namely at $\lambda_{1}=-0.3369764, \lambda_{2}=-0.1056770, \lambda_{3}=-0.0393022$, $\lambda_{4}=+0.0098145, \lambda_{5}=+0.0370746$ and $\lambda_{6}=+0.0676765$, respectively. These solutions, corresponding to $\left(f_{0}, \lambda\right)=\left(-0.65, \lambda_{n}\right), n=1,2, \ldots, 6$ are plotted in Figure 4.

A remarkable property of the subset of solutions resulting from the Reynolds analogy is that, according to Eqs. (18), the product of the dimensionless wall temperature $\theta(0)$ and the skin friction $f^{\prime \prime}(0)$ always equals unity,

$$
\theta(0) f^{\prime \prime}(0)=1 \text {. }
$$

As a consequence, the representative points of the solutions in the characteristic parameter plane $\left\{\theta(0), f^{\prime \prime}(0)\right\}$ of the present prescribed-flux boundary value problem are all located on the branches of an equilateral hyperbola. This feature is illustrated in Figure 5, where the points $\left\{\theta(0), f^{\prime \prime}(0)\right\}$ corresponding to the $6+6=12$ solutions collected in Table 1 and Figure 3 are shown.

\section{Summary and conclusions}

The subset of solutions of the title problem which can be obtained with the aid of the Reynolds analogy for the stagnation flow $(m=1)$ of a fluid with $\operatorname{Pr}=1$, has been investigated in a close relationship with the solutions reported by Merkin 


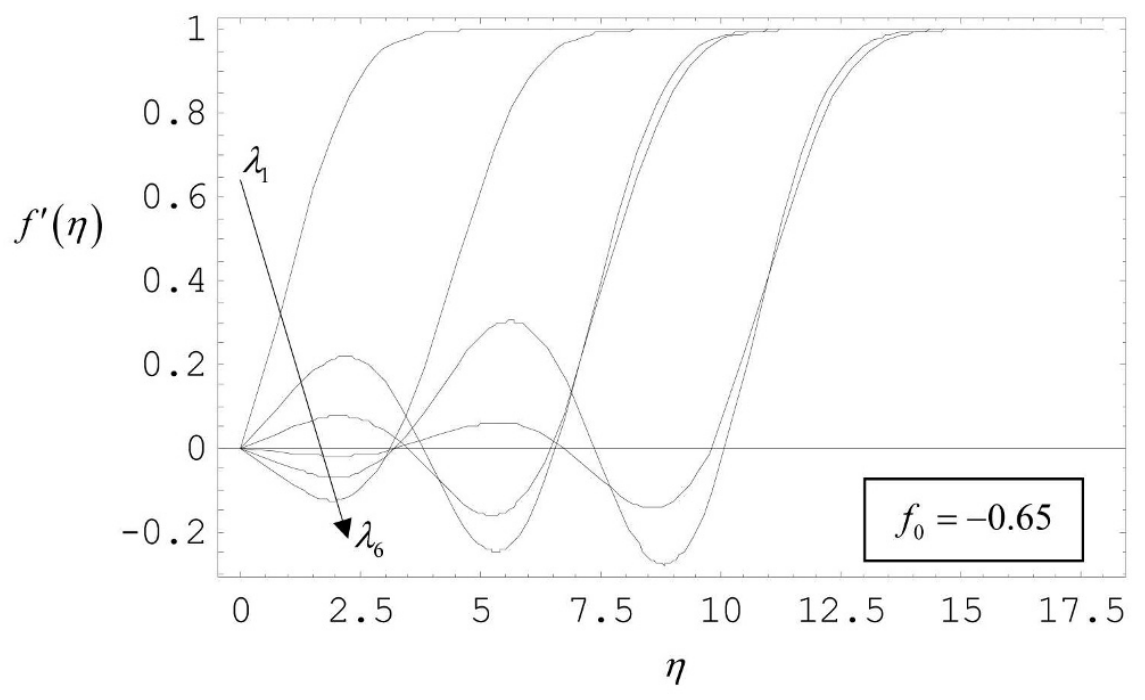

Figure 4. Plot of similar velocity profiles $f^{\prime}(\eta)$ corresponding to the skin frictions $f^{\prime \prime}(0)=-\lambda_{n}$, $n=1,2, \ldots, 6$, associated with the six $\lambda$-values specified in Figure 3 and the same value $f_{0}=-0.65$ of the injection parameter.

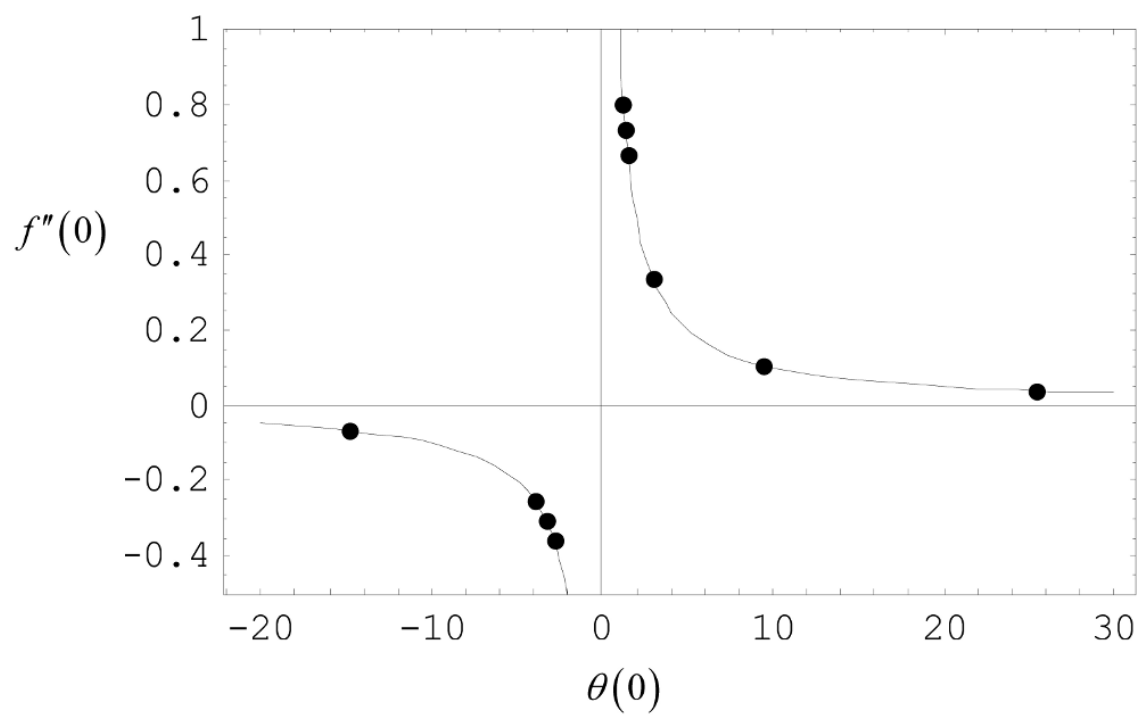

Figure 5. The representative points corresponding to the $6+6=12$ solutions collected in Table 1 and Figure 3 are all located on the branches of an equilateral hyperbola $\theta(0) f^{\prime \prime}(0)=1$. 
and Mahmood [1] and more recently by Ishak et al. [2]. Our main result shows that the representative points of this subset of solutions in the parameter plane $\left\{\theta(0), f^{\prime \prime}(0)\right\}$ are always located on the branches of an equilateral hyperbola. The Reynolds analogy-approach applies both to the assisting and opposing mixed flow regimes. Among the solutions gained in this way, flow profiles with and without backflow regions were found. The mathematical advantage of the Reynolds analogy consists of the fact that it decouples the mixed convection momentum and energy balance equations by a linear relationship between the temperature field $T$ and the streamwise velocity component $u$ of the flow.

\section{Acknowledgement}

We are indebted to a reviewer for valuable suggestions concerning the asymptotic limit of massive suction.

\section{References}

[1] J. H. Merkin and T. Mahmood, Mixed convection boundary layer similarity solutions: prescribed wall heat flux, J. Appl. Math. Phys (ZAMP) 40 (1989), 51-68.

[2] A. Ishak, J. H. Merkin, R. Nazar and I. Pop, Mixed convection boundary layer flow over a permeable vertical surface with prescribed wall heat flux, J. Appl. Math. Phys (ZAMP) 59 (2008), 100-123.

E. Magyari

Institut für Hochbautechnik

ETH Zürich

Wolfgang-Pauli Str.1

CH-8093 Zürich

Switzerland

e-mails: magyari@hbt.arch.ethz.ch and magyari@bluewin.ch

I. Pop

Faculty of Mathematics

University of Cluj

R-3400 Cluj, CP 253

Romania

(Received: November 1, 2007; revised: February 29, 2008)

Published Online First: October 10, 2008

To access this journal online:

www.birkhauser.ch/zamp 